\title{
Analytical study of idealized two-dimensional cellular detonations
}

\author{
X.Y. Hu, D.L. Zhang, Z.L. Jiang \\ Laboratory of High Temperature Gasdynamics, Institute of Mechanics, Chinese Academy of Sciences, 100080 Beijing, \\ P.R. China
}

Received 13 February 2001 / Accepted 2 August 2001

\begin{abstract}
In this study, the idealized two-dimensional detonation cells were decomposed into the primary units referred to as sub-cells. Based on the theory of oblique shock waves, an analytical formula was derived to describe the relation between the Mach number ratio through triple-shock collision and the geometric properties of the cell. By applying a modified blast wave theory, an analytical model was developed to predict the propagation of detonation waves along the cell. The calculated results show that detonation wave is, first, strengthened at the beginning of the cell after triple-shock collision, and then decays till reaching the cell end. The analytical results were compared with experimental data and previous numerical results; the agreement between them appears to be good, in general.
\end{abstract}

Key words: Gaseous detonation, Detonation cell, Cellular structure, Dynamic mechanism

\section{Introduction}

It is well known that gaseous detonation waves have threedimensional (3D) cellular structure. The tracks of cellular structure can be recorded with smoked foils on channel walls, and the regions enclosed by the tracks are called detonation cells. For extremely regular cellular patterns, such as rectangular and planar modes, which can be approximately treated as two-dimensional, it was found that the interaction of Mach configurations plays the main role. The Mach stems and incident waves of the Mach configurations alternate each other (Strehlow 1968; Fickett and Davis 1979; Nettleton 1987).

During the 70's and 80's the investigations on the propagation of detonation waves had conclusively established that detonation velocity fluctuates periodically from 0.6 to 1.5 times the C-J value (Fickett and Davis 1979; Crooker 1969; Lee 1984; Lee 1991; Mitrofanov 1996). Lundstrom and Oppenheim (1969), Strehlow (1970, 1971), Strehlow et al. (1972), Strehlow and Crook (1974) and Urtiew (1976) analyzed two-dimensional cellular detonation waves and found that the detonation velocity and pressure reach their maximum values just after the tripleshock collision, and then decay continuously until their minimum values are reached at the end of the detonation cell. However, the pressure fluctuations along the cell centerline, obtained in the experiments of VMT (Voitsekhovsky et al. 1963), suggested that detonation states close to the collision point could not be resolved because the area is too small to put in a probe. The axial velocity history recorded by Takai et al. (1974) showed a

Correspondence to: X.Y. Hu

(e-mail: xyhu@imech.ac.cn) local maximum near the apex of the cell but its accuracy is difficult to estimate. In order to explain the velocity and pressure fluctuations, Barthel (1972) suggested that a reactive compression wave near the collision point could catch up and strengthen the detonation leading front. Recently, the two-dimensional numerical results reported by Oran et al. (1998) and Gamezo et al. (1999) also showed that the leading front is initially strengthened to its maximum value at the beginning of the detonation cell.

Inspired by the insights of the previous experimental results and numerical simulations, the objective of this paper is to develop a two-dimensional analytical model to describe more exactly the triple-shock collision and propagation of detonation front through detonation cells.

\section{Regular detonation cell and its sub-cells}

The idealized two-dimensional detonation wave has a regular pattern, so that any two adjacent triple-shock structures may represent the whole front. While the leading front propagates from position I to position II, as shown in Fig. 1, any two triple-shock structures connected by an incident shock wave approach each other and then collide at the cell centerline. After the collision, the two original Mach stems now become incident shock waves and a new Mach stem is created, which connects the two newlydeveloped triple-shock structures moving apart from each other. In this way, Mach stems and incident shock waves exchange their roles and then propagate till the next collision. Meanwhile, the triple points with high pressure trace out detonation cells. Schematic of this process is shown in Fig. 1. It is easy to observe that leading fronts at the same positions relatively to different cells have the same states. 


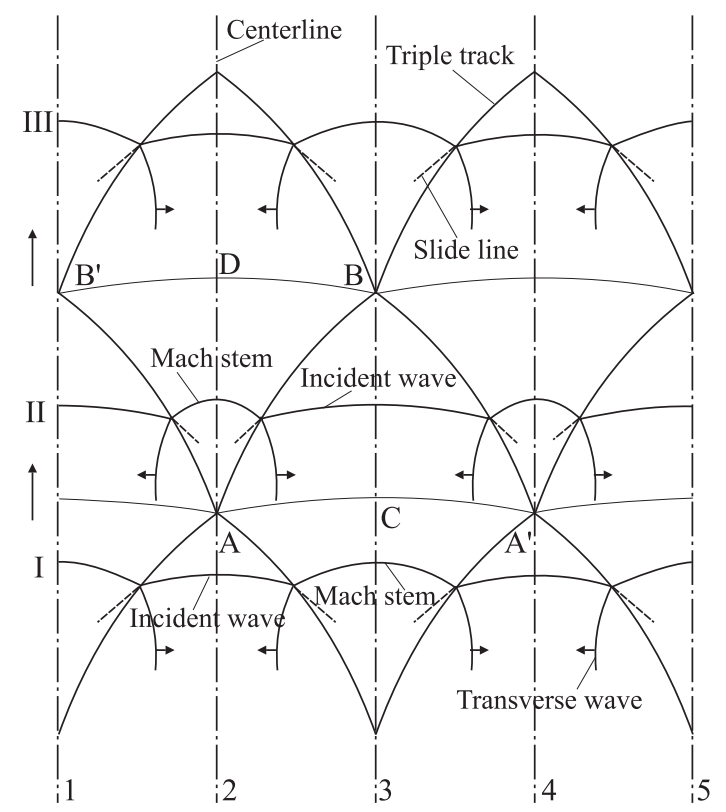

Fig. 1. Schematic of detonation cells and sub-cells: I, II, III leading front at different positions; 1, 2, 3, 4, 5-centerlines of cells; A, A', B, B' - collision points at two adjacent cells; C, $\mathrm{D}$ - intersection points of leading fronts and cell centerlines. Leading waves before and after A-C-A' (or B-D-B') are Mach stems and incident waves, respectively

Thus, the whole detonation front can be understood by analyzing the wave process taking place in one cell. Furthermore, the four track segments of a cell are similar to each other, so it is possible to decompose a cell into smaller primary units.

Based on the above discussion, a smaller primary unit called sub-cell, for instance, area $A-C-B-D$ in Fig. 1, can be defined. The sub-cell is enclosed by two centerlines (such as 2 and 3 in Fig. 1) and two leading fronts (transforming from Mach stem to incident shock wave, such as $A-C-A^{\prime}$ and $B^{\prime}-D-B$ in Fig. 1) of two adjacent cells. One can find, if the whole leading front is divided into segments by centerlines, that the sub-cell just corresponds to one dynamical cycle of a front segment. Some features of the sub-cell can be easily derived:

- The width of a sub-cell is $d / 2$, where $d$ is the cell width. The detonation cell length $l$ is related to the sub-cell dimensions as follows:

$$
A D>l / 2>C B, \quad A D+C B=l ;
$$

- The leading fronts at the two lengthways borders have the same state, so that

$$
M_{A-C-A^{\prime}}=M_{B-D-B^{\prime}}
$$

where $M_{B-D-B^{\prime}}, M_{B-D-B^{\prime}}$ are Mach numbers of the two fronts;

- If a sub-cell is divided into two parts along the triplepoint track, considering the symmetry of a detonation cell, one can find that the part with the concave triplepoint track (for instance, the area marked by $A-B-D$ in Fig. 1) stands for the first half of the detonation cell, and the other part (for instance, the area marked by $A-C-B$ in Fig. 1) stands for the second half of the cell.

\section{Triple-shock collision}

Strehlow et al. (1972), Oppenheim et al. (1972) and Urtiew (1976) investigated the problem of triple-shock collision. In their works, assuming that the incident angle $\theta_{1}$ (the angle between the incident shock wave and the triplepoint track) is invariant throughout the whole interaction, the relation between two trajectory angles, the exit angle $2 \alpha$ and the entrance angle $2 \beta$, is given as

$$
\alpha=\beta-\Delta,
$$

where $\Delta$ is the angle between the Mach stem and incident shock wave at the collision point. The relation between the exit Mach number, the entrance Mach number and the incident angle can be written as

$$
\frac{M_{\alpha}}{M_{\beta}}=\frac{\sin ^{2}\left(\theta_{1}+\Delta\right)}{\sin ^{2} \theta_{1}} .
$$

However, in most of the previous studies, only smoked foil pictures were obtained from experiments, and it is difficult to extract the incident angle from them. Therefore, it is difficult to get $M_{\alpha} / M_{\beta}$ from the cell tracks data only.

From the sub-cell properties mentioned above, it can be found that the initial and final states of triple-shock collision correspond to the states at the end point and at the beginning of a segment of triple-point track. Considering the triple-shock structures at the two ends, $A$ and $B$, of the track of a sub-cell, as shown in Fig. 2, the relation between the two states can be written as

$$
M_{A 1}=M_{B 2} ; \Delta_{A}=\Delta_{B}=\Delta ; \theta_{A 1}=\theta_{B 1}=\theta_{1},
$$

where $M_{A 1}$ is the normal shock Mach number of the incident shock wave $A C$, at point $A ; \Delta_{A}$ is the angle between the incident shock wave $A C$ and the Mach stem $A E ; \theta_{A 1}$ is the incident angle of the incident shock wave $A C ; M_{B 2}$ is the normal shock Mach number of the Mach stem $B D$ at point $B ; \Delta_{A 1}$ is the angle between the Mach stem $B D$ and the incident shock wave $B F ; \theta_{B 1}$ is the incident angle of the incident shock wave $B F$.

According to the oblique shock theory, parameters of the Mach reflection at point $A$ are expressed as follows:

$$
\begin{array}{r}
M_{i}=\frac{M_{A 1}}{\sin \theta_{A 1}}=\frac{M_{\alpha}}{\sin \theta_{A 2}}, \\
\theta_{A 1}+\theta_{A 2}=\pi-\Delta_{A}, \\
\alpha=\theta_{A 2}-\frac{\pi}{2},
\end{array}
$$

where $M_{i}$ is the inflow Mach number at point $A ; \theta_{A 2}$ is the incident angle of the Mach stem $A E$. 


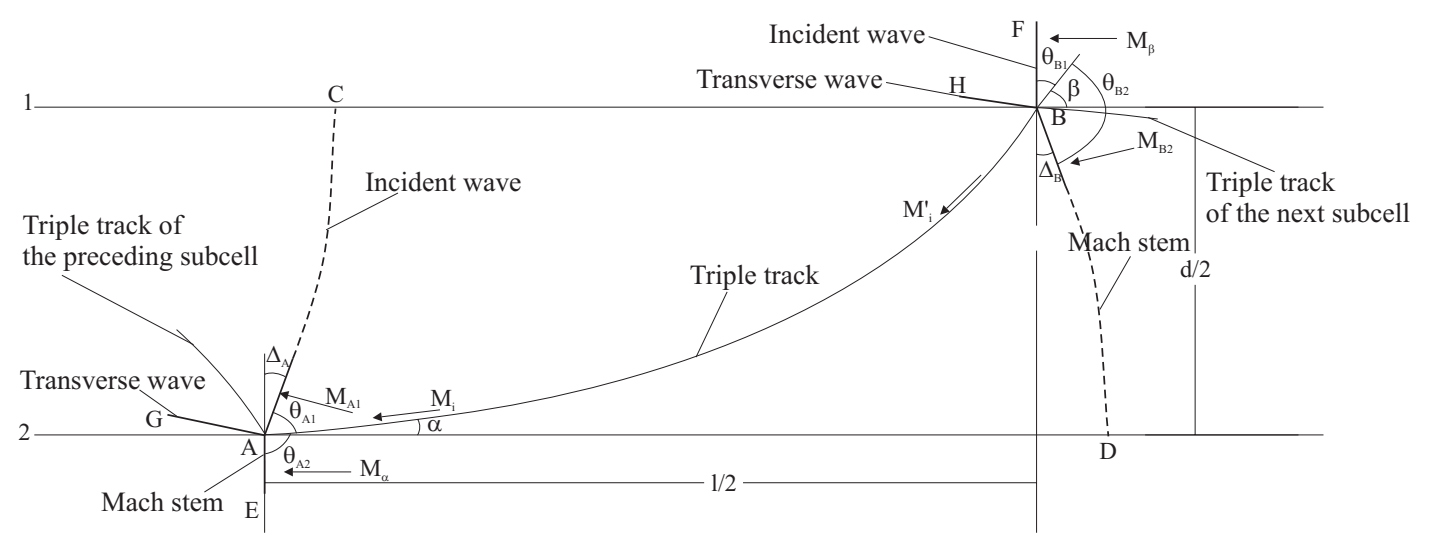

Fig. 2. Triple-shock structures at the two ends of the triple-point track of a sub-cell

Similarly, the relations for the Mach reflection at point $B$ are as follows:

$$
\begin{array}{r}
M_{i}^{\prime}=\frac{M_{\beta}}{\sin \theta_{B 1}}=\frac{M_{B 2}}{\sin \theta_{B 2}}, \\
\theta_{B 1}+\theta_{B 2}=\pi-\Delta_{B}, \\
\beta=\frac{\pi}{2}-\theta_{B 1},
\end{array}
$$

where $M_{i}^{\prime}$ is the inflow Mach number at point $B ; \theta_{B 2}$ is the incident angle of the Mach stem $B D$.

Substituting Eqs. (5) into Eqs. (7) and (8) and combining them, one may get

$$
\begin{gathered}
\frac{M_{A 1}}{M_{\alpha}}=(\operatorname{ctg} \Delta-\operatorname{tg} \alpha) \sin \Delta ; \\
\frac{M_{B 2}}{M_{\beta}}=(\operatorname{ctg} \Delta+\operatorname{tg} \beta) \sin \Delta ; \\
\frac{M_{A 1}}{M_{\alpha}}=\frac{M_{\beta}}{M_{B 2}} .
\end{gathered}
$$

Combining Eqs. (8) and (9), one may obtain

$$
\frac{M_{\beta}}{M_{\alpha}}=\frac{\operatorname{ctg} \Delta-\operatorname{tg} \alpha}{\operatorname{ctg} \Delta+\operatorname{tg} \beta} .
$$

Substituting Eq. (10) into Eqs. (8) and (9), the relation between the two trajectory angles can be reduced, that is Eq. (3). Then Eq. (11) can be written as

$$
\frac{M_{\beta}}{M_{\alpha}}=\frac{\operatorname{ctg}(\beta-\alpha)-\operatorname{tg} \alpha}{\operatorname{ctg}(\beta-\alpha)+\operatorname{tg} \beta} .
$$

Thus, using sub-cell properties and oblique shock theory, the analytical formula relating $M_{\alpha} / M_{\beta}$ and the geometry of detonation cells is developed.

With the shock wave relations and gaseous equation of state, the strength of transverse waves can be calculated from Eqs. (8) or (9). The angle $\theta_{1}$ can also be obtained by solving linked Eqs. (12) and (4). It can be concluded that $\theta_{1}$ at the collision point is not independent from the cell geometry.

The dependence of $M_{\alpha} / M_{\beta}$ on $2 \alpha$ and $2 \beta$ (Eq. (12)) is shown in Fig. 3. The average value of the entrance angle is

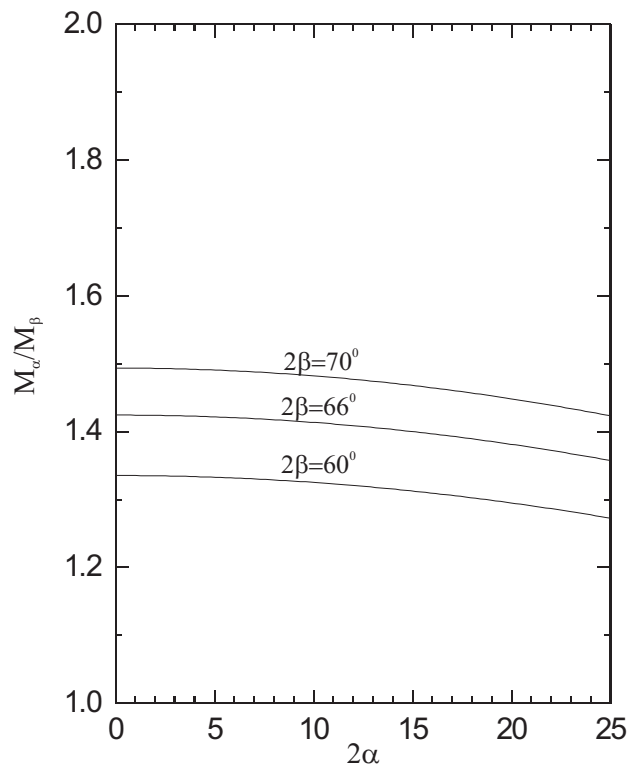

Fig. 3. Dependence of the ratio $M_{\alpha} / M_{\beta}$ from the entrance angle $2 \beta$ and the exit angle $2 \alpha$

nearly the same for all ordinary detonation systems, and appears to be very close to $70^{\circ}$ (Strehlow et al. 1972). It can also be found from Fig. 3 that $M_{\alpha} / M_{\beta}$ depends on the entrance angle to a greater extent than on the exit angle. In most cases, $2 \alpha$ is much smaller than $2 \beta ; M_{\alpha} / M_{\beta}$ is about 1.5 for almost all systems; and the corresponding $\theta_{1}$ is about $50^{\circ}$. For the $\mathrm{H}_{2} / \mathrm{O}_{2} / \mathrm{Ar}$ mixture, the exit angle is about $22^{\circ}$. The angle $\theta_{1}$ predicted with our model is about $51^{\circ}$ and $M_{\alpha} / M_{\beta}$ is 1.43 . The calculated incident angle agrees with experiments (Voitsekhovsky et al. 1963; Edward et al. 1966).

If the propagation process of detonation front through a cell is considered as a decaying process, the values of $M_{\alpha} / M_{\beta}$ predicted here is smaller than in experiments. It can be proven that the present triple-shock collision model is equivalent to the models proposed by Strehlow, Oppenheim and Urtiew. However, their solution is based on graphic techniques and does not give a relation between $\theta_{1}$ and $\alpha$ and $\beta$. In Urtiew's work on $M_{\alpha} / M_{\beta}$ calculated by Eq. (4), the incident shock angle was modified to fit 
Table 1. Comparison of Mach number ratio and incident angle

\begin{tabular}{ccc}
\hline & $M_{\alpha} / M_{\beta}$ & $\theta_{1}$ \\
\hline Urtiew (1976) & 2.85 & $36.4^{\circ}$ \\
This paper & 1.43 & $51^{\circ}$ \\
Experiments of VMT (1963) & - & about $50^{\circ}$ \\
\hline
\end{tabular}

$M_{\alpha} / M_{\beta}$ from experiments. From the foregoing discussion, $\theta_{1}$ is not an independent variable, so that it is easy to explain why $\theta_{1}$ given by Urtiew is smaller than experimental results, as shown in Table 1.

In the next section, the propagation of detonation front along a cell will be discussed in detail to show the effect of the chemical reaction induced by triple-shock collision, which results in more complex process than merely decaying one.

\section{Dynamic process in a cell}

In order to predict the development of detonation front through detonation cells, the leading front in a cell needs to be carefully modeled. In Lundstrom and Oppenheim (1969), Edward et al. (1970) and Urtiew (1976) works, the geometry of each detonation cell is associated with two extreme values of the axial velocity of the leading front. The decay factor $\lambda$ is used to account for the wave decay from one extreme to another. In the first half of the cell, the shock wave is a Mach stem while it is an incident shock wave in the second half. They defined the two-dimensional decay factor as

$$
\lambda=\frac{\mathrm{d} \ln \left(M^{-2}\right)}{\mathrm{d} \ln r},
$$

where $M$ is the normal shock Mach number of a detonation front, and $r$ is the position of the detonation front relatively to the hypothetical origin of cylindrical blast wave, which is at a certain distance $r_{0}$ from a collision point, say the apex of the cell.

Let us assume that the chemical reaction, induced by triple-shock collision near detonation cell apex, results in a concentric cylindrical compression wave that propagates forward and catches up the leading front later in the first half of the cell. So, the leading front of a cell is modeled in this paper as follows. The whole propagation of the front can be divided into two parts. The first part is from the apex to a distance $k$ with the decay factor $\lambda_{1}$, which takes into account the effect of the concentric cylindrical compression waves. The second part is the rest of the process with the decay factor $\lambda_{2}$. Both decay factors stay constant through the cell. Figure 4 shows the modified blast wave model in a sub-cell. According to the definition of sub-cell and its properties, the incident shock wave stands for the leading front in the second half of the cell, which decays from the hypothetical origin of the preceding sub-cell; the Mach stem stands for the leading front in the first half of the cell, which decays from the hypothetical origin of this sub-cell.
Using the geometric relation of the leading front, the angle between incident shock waves and Mach stems at the two ends of the triple-point track can be related to the size of the sub-cell:

$$
\Delta=\operatorname{arctg}\left(\frac{d / 2}{r_{0}+l / 2}\right) .
$$

The decay radius of blast waves at the starting point of the triple-point track is given as

$$
r_{h}=\left[\left(r_{0}+l / 2\right)^{2}+(d / 2)^{2}\right]^{1 / 2} .
$$

The decay radius at the final point of the triple-point track is $r_{0}+l$, so that the normal shock Mach number ratio between incident shock waves at point $A$ and point $B$ can be written as

$$
\frac{M_{A 1}}{M_{\beta}}=\left(\frac{r_{0}+l}{r_{h}}\right)^{\lambda_{2} / 2} .
$$

Similarly, the Mach stem decays from the apex after propagating a distance $k$, and one may obtain

$$
\frac{M_{k}}{M_{\alpha}}=\left(\frac{r_{0}}{r_{0}+k}\right)^{\lambda_{1} / 2}
$$

Note that the decay radius of Mach stems in a sub-cell at point $B$ is also $r_{h}$. Then the Mach number at distance $k$ and point $B$ can be expressed as

$$
\frac{M_{k}}{M_{B 2}}=\left(\frac{r_{0}+k}{r_{h}}\right)^{\lambda_{2} / 2} .
$$

Combining Eqs. (16), (17) and (18) with Eq. (2) results in

$$
\frac{M_{\alpha}}{M_{\beta}}=\left(\frac{r_{0}+k}{r_{0}}\right)^{\lambda_{1} / 2} \cdot\left(\frac{r_{0}+l}{r_{0}+k}\right)^{\lambda_{2} / 2} .
$$

Combining Eq. (12) with Eq. (19) yields

$$
\frac{\operatorname{ctg}(\beta-\alpha)-\operatorname{tg} \alpha}{\operatorname{ctg}(\beta-\alpha)+\operatorname{tg} \beta}=\left(\frac{r_{0}+k}{r_{0}}\right)^{\lambda_{1} / 2} \cdot\left(\frac{r_{0}+l}{r_{0}+k}\right)^{\lambda_{2} / 2} \text {. }
$$

Using Eqs. (2), (3), (9) and (14), $\lambda_{2}$ and $r_{0}$ can be calculated with the following equations using given experimental parameters $\alpha, \beta, l$ and $d$ :

$$
r_{0}=\frac{d-l \operatorname{tg}(\beta-\alpha)}{2 \operatorname{tg}(\beta-\alpha)}
$$

and

$$
\lambda_{2}=\frac{2 \ln [(\operatorname{ctg}(\beta-\alpha)+\operatorname{tg} \beta) \sin (\beta-\alpha)]}{\ln [(\operatorname{ctg}(\beta-\alpha)+l / d) \sin (\beta-\alpha)]} .
$$




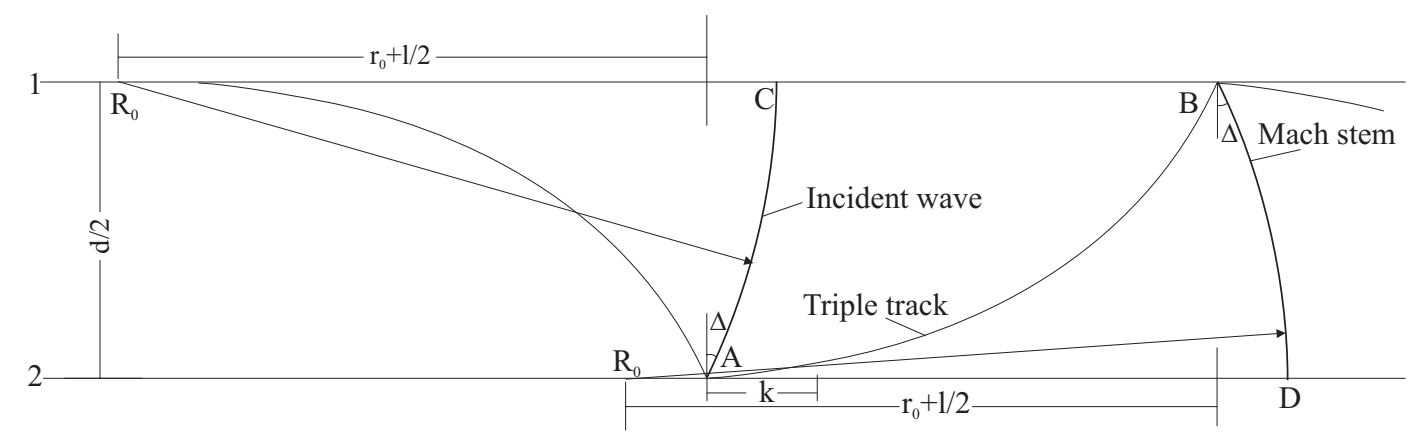

Fig. 4. Modified blast wave model in a cell

Let $M_{a v}$ represents the average Mach number of detonation fronts. One can specify the conditions for wave decay so that the total time for the decaying detonation front to pass through the sub-cell would be equal to the time needed for a steady wave at $M_{a v}$ to travel the same distance $l$, say the cell length. On the other hand, from the properties of sub-cells one can also find out that the detonation front propagation takes the same time in each half of a cell. That is

$$
\int_{r_{0}}^{r_{0}+k} \frac{d x}{M}+\int_{r_{0}+k}^{r_{h}} \frac{d x}{M}=\int_{r_{h}}^{r_{0}+l} \frac{d x}{M}=\frac{l}{2 M_{a v}} .
$$

Integrating the second part of Eq. (23) yields

$$
M_{\beta}=\frac{2 M_{a v}\left(r_{0}+l\right)}{l\left(\lambda_{2} / 2+1\right)}\left[1-\left(\frac{r_{h}}{r_{0}+l}\right)^{\lambda_{2} / 2+1}\right] .
$$

Substituting Eq. (19) into the first part of Eqs. (23), one may obtain

$$
\begin{aligned}
& \frac{\left(\lambda_{2} / 2+1\right)\left[\left(r_{0}+k\right)^{\lambda_{1} / 2+1}-r_{0}^{\lambda_{1} / 2+1}\right]}{\left(\lambda_{1} / 2+1\right)\left(r_{0}+k\right)^{\left(\lambda_{1}-\lambda_{2}\right) / 2}}= \\
& =\left[\left(r_{0}+k\right)^{\lambda_{2} / 2+1}+\left(r_{0}+l\right)^{\lambda_{2} / 2+1}-2 r_{h}^{\lambda_{2} / 2+1}\right] .
\end{aligned}
$$

The values of $r_{0}, r_{h}, \lambda_{2}, M_{\beta}$ and $M_{\alpha}$ can be calculated directly from Eqs. (21), (15), (22), (24) and (12). By solving linked Eqs. (20) and (25) numerically, $\lambda_{1}$ and $k$ can be also obtained. Now, having two trajectory angles, the cell size from given smoke foil data and $M_{a v}$, all parameters governing the propagation of leading front can be determined.

Using decay factors $\lambda_{1}$ and $\lambda_{2}$, and Mach numbers of the leading front at the two ends of a cell $M_{\alpha}$ and $M_{\beta}$, the detonation velocity at the distance $k$ from the apex is written as:

$$
M=M_{\alpha}\left(\frac{R}{R+x}\right)^{\lambda_{1} / 2}, 0 \leq x \leq k / l,
$$

where $R=r_{0} / l$ is the normalized distance of the hypothetical origin from the apex, $x$ is the normalized distance through the cell.
In the rest of the cell

$$
M=M_{\beta}\left(\frac{R+1}{R+x}\right)^{\lambda_{2} / 2}, \quad k / l \leq x \leq 1 .
$$

With the gaseous equation of state, detonation states in a cell can also be derived from Eqs. (26) and (27), which include detonation pressure, transverse wave strength etc.

For smoked foil data of a $\mathrm{H}_{2} / \mathrm{O}_{2} / \mathrm{Ar}$ mixture, $\lambda_{1}$ and $\lambda_{2}$ calculated from our blast model is -0.94 and 0.8 . The predicted propagation of leading fronts along the centerline in comparison with experiments is shown in Fig. 5. The results indicate that the dynamic process consists of two stages. First, the detonation front is strengthened near the cell apex and reaches its maximum intensity at about $0.1 l$, and then decays till the end of the cell. The decaying process calculated by our new model is in good agreement with the experiments (Strehlow and Crook 1974; Hanana et al. 2000). However, the reported second pressure jump at $0.7 l$, which is consequence of collision of two transverse waves, can not be predicted by our model. As to the detailed process near the apex, it is difficult to verify it because of the lack of experimental data. However, the numerical results reported by Oran et al. (1998) and Gamezo et al. (1999) showed that detonation velocity increases just after the triple-shock collision and the maximum is at about $0.1 l \sim 0.2 l$ away from the apex, which is in good agreement with Fig. 5.

\section{Conclusion}

In this paper, the concept of detonation sub-cells is introduced and used to describe cellular detonation. Based on the analysis of the sub-cells, an analytical formula is derived to describe the relation between the Mach number ratio through triple-shock collision and the geometric properties of detonation cell. A modified blast wave model is proposed to describe the propagation of detonation front through a cell. The predicted velocity and pressure fluctuations of the detonation front show that detonation waves are initially strengthened after triple-shock collisions at the beginning of the cell, and then decay until they reach the cell end. These analytical results were compared with experimental data and previous numerical 


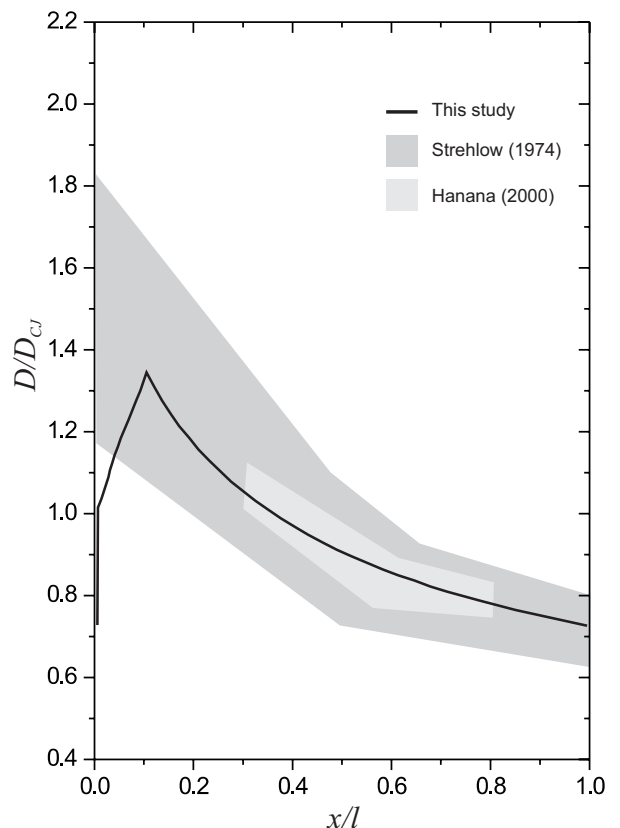

Fig. 5. Variation of detonation velocity along the centerline

results. The agreement between them appears to be good, in general.

\section{References}

Barthel HO (1972) Reaction zone-shock front coupling in detonations. Physics of Fluids 15(1):43-50

Crooker AJ (1969) Phenomenological investigation of low mode marginal planar detonations. Ph.D. Thesis, University of Illinnois

Edwards DH, Parry DJ, Jones AT (1966) On the coupling between spinning detonation and oscillation behind the wave. Br. J. Appl. Phys. 17:1507-1510

Edwards DH, Hopper G, Job EM, Parry DJ (1970) The behaviour of the frontal and transverse shocks in gaseous detonation waves. Acta Astronautica 15:323-333

Fickett W, Davis WC (1979) Detonation. University of California Press

Gamezo VN, Desbordes D, Oran ES (1999) Formation and evolution of two-dimensional cellular detonations. Combustion and Flame 116:154-165
Hanana M, Lefebvre MH, Van Tiggelen PJ (2000) Preliminary experimental investigation of the pressure evolution in detonation cells. Experimental Thermal and Fluid Science 21:64-70

Lee JHS (1984) Dynamic parameters of gaseous detonations. Ann. Rev. Fluid Mech. 16:311-336

Lee JHS (1991) Dynamic structure of detonation in gaseous and dispersed media. Kluwer Academic Publishers, pp. 125

Lundstrom EA, Oppenheim AE (1969) On the influence of nonsteadiness on the thickness of the detonation waves. Proc. of Roy. Soc. A 310:463-478

Mitrofanov VV (1996) Modern view of gas detonation mechanisms. Progress in Astronautics and Aeronautics 137:327340

Nettleton MA (1987) Gaseous detonation. Chapman and Hall

Oppenheim AE, Smolen, JJ, Kwak D, Urtiew PA (1972) On the dynamics of shock intersections. In: Fifth Symposium (International) on Detonation, ONR, Department of Navy, Arlington, Va, pp. 119-136

Oran ES, Weber JE, Stefaniw EI, Lefebvre MH, Anderson JD (1998) A numerical study of two-dimensional $\mathrm{H}_{2}-\mathrm{O}_{2}-$ Ar detonation using a detailed chemical reaction model. Combustion and Flames 113:147-163

Strehlow RA (1968) Gas phase detonations: recent developments. Combustion and Flame 12(2): 81-101

Strehlow RA (1970) Multi-dimensional detonation wave structure. Astronautica Acta 15:345-357

Strehlow RA (1971) Detonation structure and gross properties. Combustion Science Technology 4:65-71

Strehlow RA, Admaczyk AA, Stiles RJ (1972) Transient studies of detonation waves. Acta Astronautica 17:509-527

Strehlow RA, Crook AJ (1974) The structure of marginal detonation waves. Acta Astonautica 1:303-315

Takai R, Yoneda K, Hikita T (1974) Study of detonation wave structure. In: Proc. Fifteenth Symposium (International) on Combustion, pp. 68-78

Urtiew PA (1976) Idealized two-dimensional detonation waves in gaseous mixtures. Acta Astronautica 3:187-200

Voitsekhovsky BV, Mitrofanov VV, Topchian ME (1963) Front structure of detonation in gases. Izdatel'stvo Sibirskogo Otdeleniya AN SSSR (Publishing of Sibirean Branch of the USSR Academy of Sciences) 